\title{
Note by Editor
}

The makers of the Logicon Caries Detector ${ }^{\mathrm{TM}}$ system, which was the subject of a recent paper in this journal [Wenzel A: Computer-automated caries detection in digital bitewings: Consistency of a program and its influence on observer agreement. Caries Res 2001;35:12-20], point out that further information on the performance of the system can be found in the material supplied to the Food and Drug Administration. This material is available at http://www.fda.gov/cdrh/pma/pmasep98.html (go to P980025).

\section{Author Index Vol. 35, 2001}

Abstracts of the 48th ORCA Congress (issue No. 4) have their own Author Index

Addy, M. 61, 211

Akagi, T. 47

Alaluusua, S. 36, 390

Alanen, P. 173

Ando, M. 464

Angmar-Månsson, B. 21

Aps, J.K.M. 407

Attin, T. 216

Balakrishnan, M. 75

Balz, M. 142

Bánóczy, J. 167

Baysan, A. 41

Beighton, D. 3, 8, 397, 421

Berkowitz, J. 95

Birkeland, J.M. 162

Bizhang, M. 156

Bjorvatn, K. 162

Bogaerts, K. 442

Boros, I. 167

Borsboom, P. 41

Bosch, J.J. ten 1

Bowen, W.H. 67, 331
Brailsford, S.R. 397, 421

Brookes, S.J. 50, 136

Buchalla, W. 216

Campus, G. 427

Castiglia, P. 427

Cate, J.M. ten 317

Chow, L.C. 116, 223

Claeys, G. 407

Clark, D.C. 95

Cohen Carneiro, F. 384

Cury, J.A. 106

Davies, R. 41

Davis, B.A. 331

Dawes, C. 344

Declerck, D. 442

Del Bel Cury, A.A. 106

Dibdin, G.H. 344

Dolan, T.A. 366

Drummond, B.K. 235

Duncan, R.P. 366
Ebisu, S. 111

Eisenburger, M. 61, 211

Ellwood, R. 41

Eschrich, K. 258

Fan, M.W. 458

Foerster, U. 366

Frencken, J.E. 90

Ganss, C. 325

Gest, P.E. 149

Gestalin, A. 149

Gilbert, G.H. 366

Haak, R. 193

Haftenberger, M. 451

Hahn, P. 205

Hannig, M. 142

Hashizume, L.N. 106

Hata, S. 338

Hatakka, K. 412

Haugejorden, O. 162

Hausen, H. 360
Helenius, H. 163, 435

Heller, K. 376

Hellwig, E. 205

Hetzer, G. 451

Hintze, H. 27

Hof, M.A. van’t 90, 162, 186

Hughes, J.A. 61, 211

Huovinen, P. 163

Huysmans, M.C.D.N.J.M. 83

Hyssälä, L. 435

Isokangas, P. 173

Isotupa, K.P. 129

Iwami, Y. 111

Jälevik, B. 390

Julihn, A. 178

Kage, A. 156

Kärkkäinen, S. 360

Keels, M.A. 376

Keszler, P. 167

Ketley, C.E. 252

\section{KARGER}

(C) 2001 S. Karger AG, Basel

Fax + 41613061234 E-Mail karger@karger.ch www. karger.com
Accessible online at

www. karger.com/journals/cre 
Kidd, E.A.M. 3, 8, 421

Kimura, T. 47

Kinoshita, T. 47

Kirkham, J. 50, 136

Kivilompolo, T. 129

Klimek, J. 325

Kneist, S. 258

Knöfel, S. 216

Kopec, L.K. 67

Korpela, R. 412

Kotsanos, N. 200

Kovač-Kavčič, M. 247

Lagerweij, M.D. 317

Lai, S. 427

Larsen, M.J. 57, 229

Larsson, I. 178

Le Flohic, A.M. 149

Leistevuo, J. 163

Leistevuo, T. 163

Lennon, M.A. 252

Leppäniemi, A. 36

Lesaffre, E. 442

Levy, S.M. 95

Liao, H. 223

Lo, E.C.M. 458

Loveren, C. van 186

Luke, J. 125

Lukinmaa, P.-L. 36

Lumbau, A. 427

Luo, Y. 458

Lynch, E. 41

McIver, F.T. 376

Mäkinen, K.K. 129

Mäkinen, P.L. 129

Mandari, G.J. 90

Maragakis, G.M. 205

Marchant, S. 397
Martens, L.C. 407, 442

Mattila, M.-L. 435

Maupomé, G. 95

Mayanagi, H. 338

Mejàre, I. 178

Meldrum, A.M. 235

Merte, K. 258

Meurman, J.H. 412

Moalic, E. 149

Morita, M. 47

Mukai, Y. 317

Nadanovsky, P. 384

Nair, M.K. 354

Nair, U.P. 354

Näse, L. 412

Neumeister, V. 451

Ng-Evans, L. 67

Noack, M.J. 193

Nunn, M.E. 240

Ojanlatva, A. 435

Österblad, M. 163

Palenstein Helderman, W.H. van 162,186

Paunio, P. 435

Pearson, S.K. 331

Pereira, A.C. 83

Permert, L. 178

Petersson, L. 41

Pienihäkkinen, K. 173

Pönkä, A. 412

Poussa, T. 412

Pyy, L. 163

Quinio, D. 149

Quiñonez, R.B. 376
Ratledge, D.K. 3, 8

Raubertas, R.F. 331

Rautava, P. 435

Richards, A. 57

Roberts, G.J. 397

Robinson, C. 50, 136

Rupf, S. 258

Rwenyonyi, C.M. 162

Savilahti, E. 412

Saxelin, M. 412

Schäffer, U. 325

Schemehorn, B.R. 464

Schumacher, G.E. 116

Sears, M.R. 235

Seemann, R. 156

Seppä, L. 360

Sheehy, E.C. 421

Shellis, R.P. 61, 211

Shi, X.-Q. 21

Shore, R.C. 50, 136

Shulman, J.D. 95, 240

Sillanpää, M. 435

Simmonds, R.S. 75

Skalerič, U. 247

Smith, D.A. 50

Söderling, E. 129, 173

Solinas, G. 427

Souza de Mello, F. 384

Spall, T. 325

Stenlund, H. 178

Stookey, G.K. 464

Stösser, L. 258

Tabchoury, C.P.M. 106

Tagg, J.R. 75

Takagi, S. 223

Taylor, S.E. 240

Tenovuo, J. 163, 173
Thomson, W.M. 235

Toivanen, J. 129

Tomii, Y. 111

Tranæus, S. 21

Truin, G.J. 90

Tsuneishi, M. 47

Tütüncü, R. 216

Twomey, A.C. 397

Unezaki, T. 111

Vacca Smith, A.M. 67

Van Maele, G.O.G. 407

Vann, W.F., Jr. 376

Vanobbergen, J. 442

Veen, M.H. van der 464

Verdonschot, E.H. 83

Viergutz, G. 451

Vogel, G.L. 116

Wallwork, M.L. 50

Watanabe, T. 47

Weerheijm, K.L. 390

Wei, S.H.Y. 458

Wenzel, A. 12

West, N.X. 61

Whitt, J.K. 376

Wicht, M.J. 193

Wood, S.R. 50, 136

Wunder, D. 67

Yamamoto, H. 111

Yamashita, F. 47

Zerilli, A. 149

Zhang, J. 50

Zhang, Z. 116

Zimmer, S. 156

Zoitopoulos, L. 421 